\title{
Análisis al desarrollo social territorial: estimular competencias y fortalecer estrategias
}

\author{
Territorial social development analysis encourage competition and strengthening strategies
}

\author{
Cristian Eugenio Orellana Pino ${ }^{1}$
}

\begin{abstract}
Resumen
Al observar la dinámica regional este trabajo pretende evaluar la acción de los agentes sociales en el modo de aplicar los instrumentos y métodos con objetivos a retos estratégicos. Este trabajo pretende evaluar como implementar proyectos bajo la visión del control de gestión como proceso clave para alcanzar los objetivos de un plano de integración comercial; comprender la necesidad de evaluar herramientas políticas, sociales y económicas, ya que en su ausencia, pueden causar problemas en metodologías cuando aplicadas en espacios periféricos; entender el análisis de la integración de los territorios con miras a la creación de polos regionales estratégicos estableciendo escenarios que se promueven por la creación de un planificado proceso de gestión social. Ciertos países Latinoamericanos, han podido demostrar con casos concretos, que si bien planificados, pueden proyectarse a territorios periféricos en el uso de una estrategia que fortalezca el crecimiento y su desarrollo.
\end{abstract}

Palabras-Claves: Agentes sociales. Desarrollo. Excedentes territorial.

\begin{abstract}
Taking into consideration the regional dynamics, this paper evaluates the action of the social partners when using methodological tools for new strategic challenges. This paper aims to evaluate how to implement projects under the vision of management as a process to achieve the key objectives of a plan of trade integration; to stress the need to evaluate political, social and economic tools, since their absence may cause methodological problems when applied in peripheral areas; to understand the analysis of the territories integration in order to create strategic regional poles to establish scenarios to promote by the creation of a planned process of social management. Certain Latin American countries have been able to demonstrate with concrete cases that, if well planned, these strategies can be projected to peripheral areas to strengthen its growth and development.
\end{abstract}

Keywords: Agentes social. Spatial development. Surplus.

\section{Introducción}

La diferenciación entre las funciones que cada agente social desempeña como fuerza monopolizadora es crear acciones compensatorias. Según Elias (1993) estos se definen como espacio sociales pacificadores, que moderan la participación desproporcional de los grupos desarticulados, eliminando acciones emotivas individuales, para dar espacio al colectivo. Consecuentemente su dependencia a una relación organizacional

Artigo submetido em 24 de julho de 2010 e aceito para publicação em 25 de março de 2011.

1 Académico de Ingeniería en Administración de Empresas, Escuela de Ingeniera en Administración de Empresas, Finanzas $Y$ Marketing, Facultad de Economía y Empresa, Universidad Diego Portales, Santiago, Chile. Dirección: Calle Boris Bravo 9528, La Florida, RM - Santiago (Personal). E-mail: cristian1914@hotmail.com 
social de participación. El proceso civilizador conduce la acción de tareas organizadas que siguen un plano director de ejecución, este proceso natural no obedece a un modelo racional ni menos irracional. Es una respuesta a la acción conjunta de varios elementos que organizados dan un sentido lógico a su articulación por una dinámica social progresiva. No existe acción completamente aislada que lleve cambios específicos sin la intervención del entramado social. La dependencia que la fuerza monopolizadora desarrolla genera conductos competitivos y por su vez diferenciadora por las funciones que cada individuo miembro es investido. Las acciones que los grupos sociales emprenden para mejoras en los aspectos sociales, económicos, políticos, culturales, pasan por una revisión a la estructura de satisfacciones sobre los bienes y servicios. Estos dependen cada vez mas ya no del propio consumo sino que mas bien de los demás (HIRSCH, 1979).

Esta dependencia contrasta con las acciones aisladas pues necesariamente muestra que debe existir un encadenamiento de necesidades de dependencia mutua entre sociedad e institucionalidad. La colaboración y el entendimiento por ambas partes produce una efecto en la nueva organización que se ilustra de forma genérica en la relación que es necesaria para elaborar robustos proyectos de inversión (MARTINEZ, 1993). Plano, programas, proyectos que sean direccionados a fortalecer la participación entre organizaciones, mancomunales, cooperativas, redes sociales y sus pares institucionalista gubernamentales. Cada relación pueda generar grupos funcionales, empresas, entidades que formen asociaciones, conglomerados productivos, que articulen y se entiendan. Que esa dependencia generalizada se comprenda como participación en la asignación de recursos limitantes dentro de los espacios productivos, en los límites que establecen las regiones, microrregiones, localidades que ofrezcan un atractivo para la creación de procesos endógenos. Esto inserido dentro del marco regulatorio de valorización del entorno territorial; la acción conjunta entre los agentes sociales; desarrollo de lazos de mutuo intercambio para crear valor y establecer relaciones con los demás territorios y el resto del mundo, en suma que estos elementos sean útiles para dar forma y dimensión como capacidad de generar estrategias territoriales de competitividad (OBSERVATORIO EUROPEO, 1999). Como un todo, el enfoque que pretende evaluar este trabajo es diluir las etapas que llevan a comprender las relaciones entre las realidades y pretensiones institucionales y supranacionales vinculados a las necesidades territoriales de las regiones con sus ímpetus de enfrentar la realidad con nuevos enfoques.

\section{Los impactos que desencadenan la desarticulación en las acciones participativas entre sociedad y gobierno}

Según Choussudovsky (1997), la globalización de la pobreza es acompañada por la reforma de las economías nacionales de los países en desarrollo y por la redefinición del rol que cumplen en esta nueva orden económica. Frente a este panorama algunos elementos parecen relevantes y comunes pues son típicos en varias regiones de Latinoamérica: i) desempleo, ii) descomposición económica, iii) disminución de salarios, iv) retracción mundial del consumo que trae como resultado abandono o desplazamiento de unidades productivas, v) apropiación de excedentes por no productos, vi) transferencia de economías de servicio para economías de arriendo. En su totalidad los eventos en contextos particulares alteran los enfoques de la estructura, cada uno presenta una serie de situaciones que desencadenan conflictos nefastos. En su mayoría trazan fronteras donde se establecen límites sociales para nivelar el crecimiento articulado de cada uno de acuerdo a su territorio y a la capacidad de absorber la influencia negativa que estos componentes conflictivos causan. Sin dejar de lado el contexto globalizante en cual se encuentra, nacional, supranacional, territorial, regional, micro-regional; pues los efectos económicos de este proceso se manifiestan de forma diferente en cada uno. De tal manera que la configuración de cada uno, logre evitar tales eventos pues es en este contexto que la dependencia se torna bastante evidente.

Y quien lo determina es la estructura organizacional administrativa que debe ser reconfigurada, para evitar los males que la dependencia económica en países subdesarrollados genera. Erradicar los atávicos fundamentalismos del desarrollo económico cuando la estructura en regiones de subdesarrollo dispone configurar una dependencia de módulos de importación. Es deber de las organizaciones estimular el 
crecimiento procediendo por medio de reorientaciones dando paso a estudios de capacidad de cada región, eficacia manipuladora del medio ambiente natural, el parque tecnológico, el aspecto cultural-social y principalmente las unidades políticas y geográficas (ESTRELLA y VALERIO, 2006).

A finales del siglo pasado, el rol de como algunos Estados Latinoamericanos conducían las directrices económicas para establecimiento de nuevas bases de apoyo en que la institucionalidad enfocaría las responsabilidades de liberación comercial sobre emprendimientos de iniciativa privada. Algunos Estados adoptaron medidas de incentivo y apoyo bajo la visión de concursos de proyectos sobre el sistema de planos de desarrollo regional optando por propuestas mejoradas del sistema privado y consecuentemente como ejecutor de los recursos públicos (LORENTE, 2006).

La iniciativa de estimular el desarrollo regional a través financiamiento de proyectos es un estimulo que objetiva la recuperación de territorios por propuesta de integración social. Sin embargo, muchas veces el efecto no es real, debido a la inequidad por la falta de elementos transparentes que dejan de atender la igualdad entre los grupos sociales, la equidad en el acceso a las informaciones, proyectos, financiamientos, tecnologías, visualizar las ventajas de cada región por medio de sofisticados sistemas de evaluaciones, que den garantía de retorno a las propuestas, que muchas veces las empresas privadas acaban absorbiendo. Para evitar esta desarticulación entre las entidades publicas, organizaciones de pequeño, medio y grande porte, se torna necesario crear medidas de ajuste de control protocolar para accionar instrumentos de resolución de eventos, cuando el ambiente o entorno es desfavorable para los acuerdos negociados.

Entender la pluralidad de los mercados como forma de capitular las vías de comercialización para productos subyacentes de cada territorio y en conjunto que sean integrados al desarrollo sectorial categóricamente como entidades cooperadas fortaleciéndose los lazos entre productos comunes. Una vez habiendo detectado los conflictos recurrentes que llevan a la desestabilización de los programas de inserción territorial, han de minimizar las oscilaciones de las actividades económicas, que en momento puedan traer resultados funestos como: mala distribución de renta, crecimiento desproporcionado e inestabilidad económica que conlleva a una situación de inflación. Para tanto, minimizar los conflictos con el fin de obtener un resultado en cuanto al crecimiento y que este sea estimulado por la oferta, controlando los recursos naturales, recursos disponibles de trabajo, tecnología y recursos de capital social. Dándole atención a la fuente mayor de la demanda que es el consumo, según Hirsh (1979), siendo que en función del consumo se formula un sistema de evaluación que el proprio consumidor establece como estimulo a la medida económica para las actividades de inserción regional.

La incapacidad de estas inserciones regionales se ve limitada por la aridez de proyectos innovadores, orientados por una substitución de recursos que se encuentran disminuidos en sectores económicos y proyectos sostenibles que son limitados por un espacio territorial, en su conjunto estos principios se anulan sin la debida conducción para resultados de buen desempeño. Ya la Figura 1 muestra las relaciones bipolares de un Gobierno con supremo y excesivo control sobre los medios de producción social, creando acciones soberanas dentro del cuadro institucional como agente centralizador de políticas económicas, un estado que conduce a la yuxtaposición de centros de decisión política y económica (ROSSETI, 1987). La combinación de estos trazos acaba por subordinar el sistema económico y su operación como aparato gestor a varias formas de gestión centralizada, dando como natural la creación de organismos estatales, donde centralizan las iniciativas económicas y subsecuente control. 
Figura 1

\section{Características de economías colectivas e individuales de control Gubernamental de Plena y Parcial intervención}

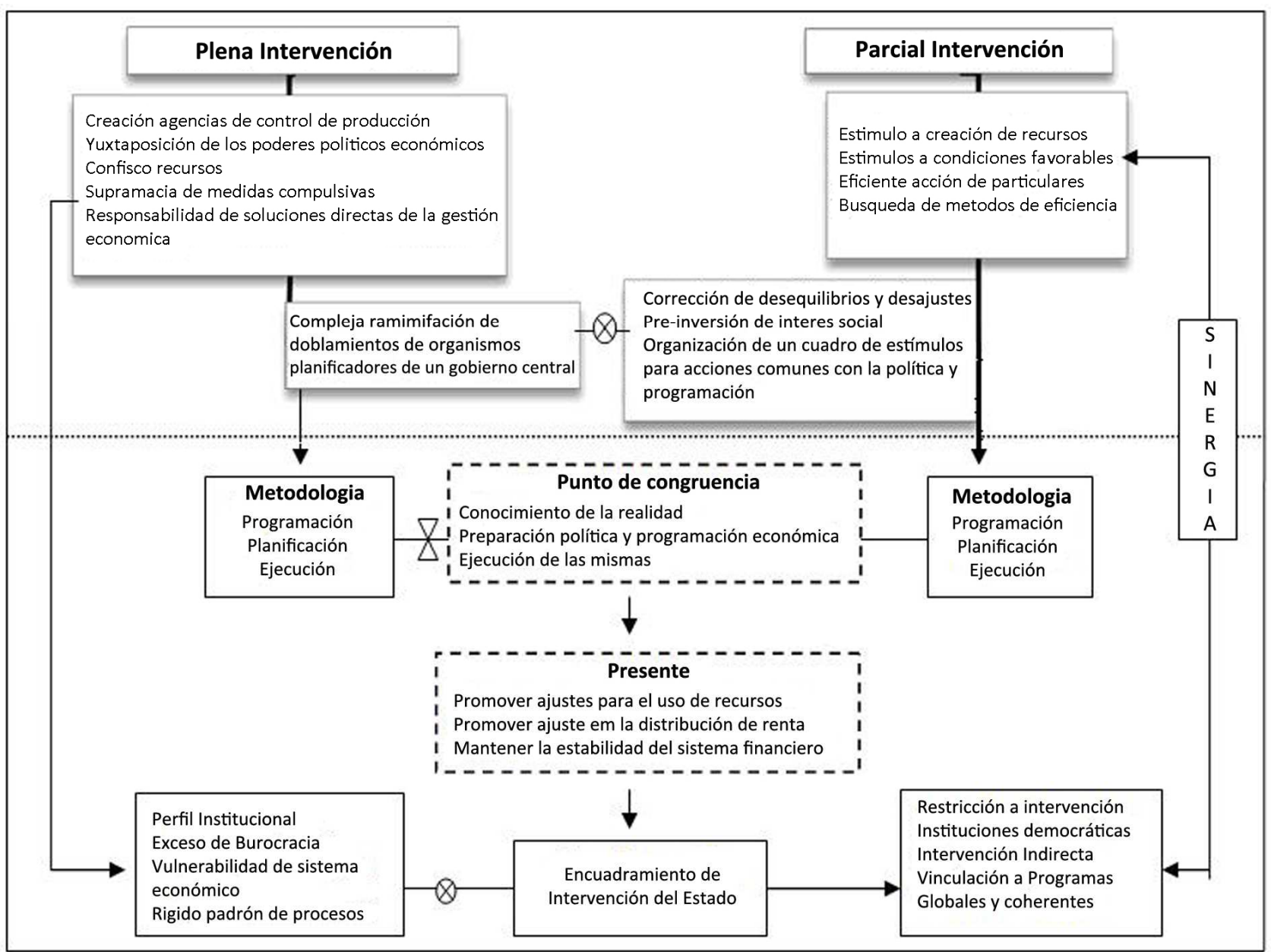

Fuente: elaborado por el autor.

En contra posición a la intervención plena del Estado, la forma extrema conceptuada como intervención Parcial, el Estado representa un organismo que necesariamente se torna un ente dilatador de actividades que soporta una mayor responsabilidad de las funciones, programas de asistencia social, seguridad, proyectos de intervención y ayuda, a medida que la complejidad del sistema crece nuevas funciones le son otorgadas. Las motivaciones según Rosseti (1987) son lo que torna imprescindible la participación parcial del papel del Estado sobre los procesos administrativos, es promover la estabilidad económica, equidad en la renta y distribución eficaz de los recursos.

Como medio de coordinar las actividades justamente en la dilatación de aquellas funciones que tornaron necesaria su presencia, entendiendo esta como la corrección de los desajustes provocados por la incapacidad de los mecanismos del libre mercado en promover durante las primeras décadas del siglo pasado, una equidad social.

Otra acción que dio respuesta a la intervención del Estado fue sustituir la iniciativa privada, sobre todo en la ejecución de emprendimientos de servicios y bienes públicos de interés social, implantación de infraestructura, prospección de recursos naturales, etcétera. La coordinación general de los agentes económicos en el pleno sentido de alcanzar los fines de la política económica en circunstancias en que la iniciativa 
privada torna necesaria las medidas políticas económicas, en el sentido de que los objetivos de crecimiento, estabilidad y mejor repartición de los resultados de las actividades sociales de producción puedan ser efectivamente cumplidos.

\section{El modelo estratégico territorial (MET) y el carácter de los indicadores a través de la matriz FODA (fortaleza, oportunidades, debilidades y amenazas).}

La simple noción cuando se examinan los obstáculos prácticos sobre la instalación de un sistema de gestión innovador aliado al uso de estrategias competitivas, dentro de un proceso de encadenamientos productivos, es dar atención al entorno inmediato de aglomeración donde se encuentra (BARROSO, 2006). Según Bettelhem (1976), sugieren que en su primera instancia se evalúen los comportamientos que desencadenan estos tipos eventos. En el ámbito de los retos que cabe a la participación de agentes sociales es urgente establecer las directrices con los cuales han de manifestarse sus necesidades sectoriales a través del MET. Con relación al desarrollo territorial descentralizado, estos vínculos suelen ser más evidentes debido a que se exponen por completo los efectos de las desigualdades cuando el retorno de los activos no se recupera en tales territorios, visto en políticas de gobierno demasiados centralizados.

Ya la importancia de esta descentralización es mucho más una cuestión de equidad social y participación ciudadana que dar énfasis a intereses mayores desproveído de sustancia en el dialogo. Esta parte del trabajo conduce la atención hacia el análisis sistemático de evaluaciones usando una matriz FODA como métodos de ajuste de control. El estudio en si caracteriza en evaluar cada territorio de acuerdo a sus capacidades técnicas que poseen, manifestando el potencial con esto aumentando las vías estratégicas que se canalizan hacia donde deben direccionarse los procesos de gestión (Figura 2). Al observar las tipologías correspondientes a los sectores envueltos, obliga a la formación sistémica de cada proceso a inserirse en los intereses regionales, al ver los input característicos del proceso suelen ser comunes a todos los estudios en cuestión el territorio el elemento mas importante envuelto en el confronto, las tipologías son las partes mediadoras y funcionales de la cadena productiva, a los output de resultados se integran a las evaluaciones que por si solo no son suficientes para el MET y las regiones, puesto que es necesaria para este examen, el análisis de cada elemento en la Matriz FODA. 
Figura 2

Modelo Estratégico Territorial (MET-FODA)

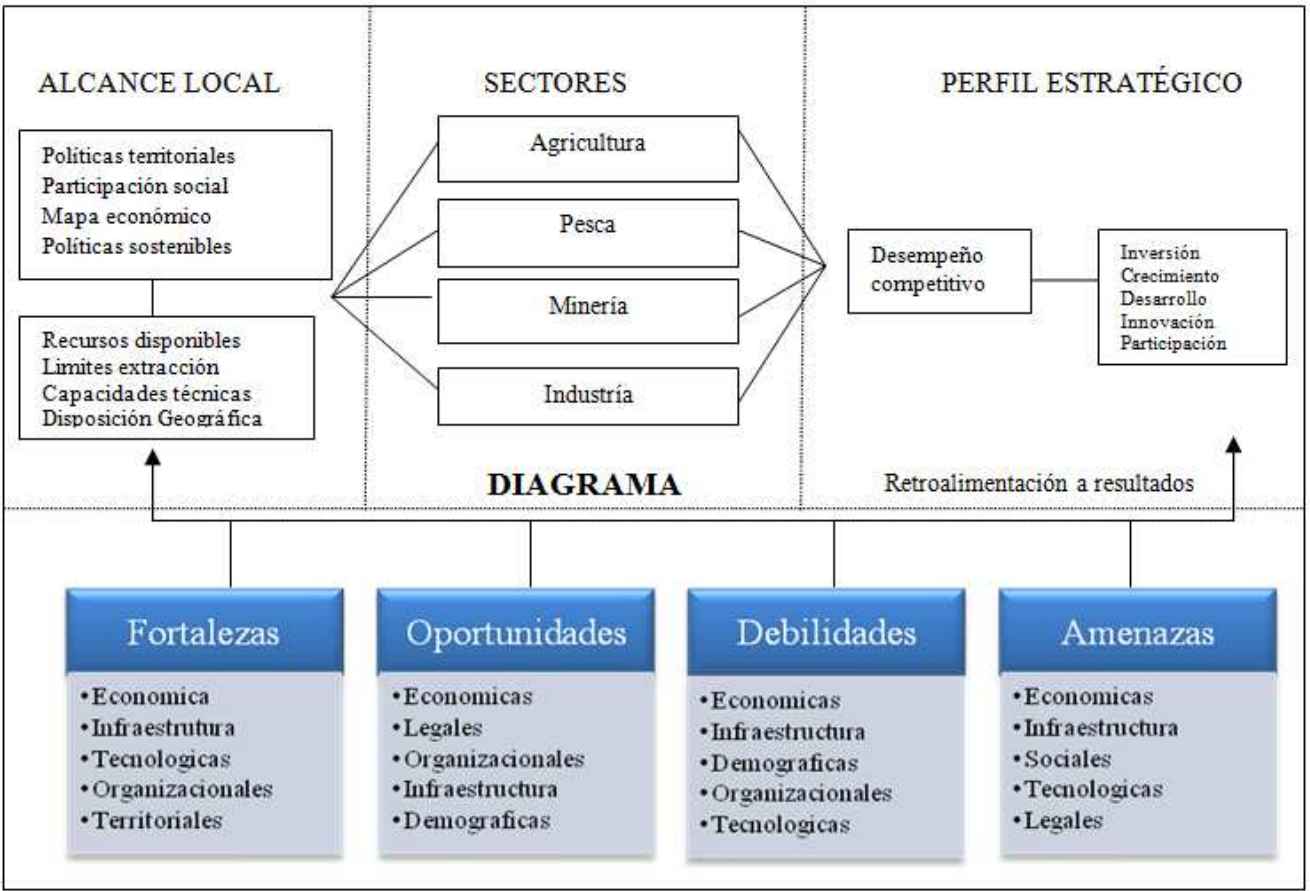

Fuente: elaborado por el autor.

El MET deberá ser claro y específico, como amplio, además de su alcance y penetración en la cadena productiva al aumentar las vías que otorgaran posibilidades mayores en abertura logística a comercializar los productos creando valores agregados. Pues el uso de economías flexibles que permita el uso de pequeña escala, dinámicas y que se acomodan con simplicidad a espacios micro-regionales, además que permiten el enraizamiento de empresas locales junto aumentar la red de organizaciones conglomeradas. Con esto se manifiestan posicionamientos diferenciados en el momento de realizar el diseño e implementar el proyecto del MET.

El proprio autor destaca que es imperativo el contacto social entre los agentes que participan en la gestión del oficio estratégico territorial, pues se esta trabajando para generar vínculos que de facilidad al intercambio de saberes y experiencias. Es un universo de entidades que hacen parte una red de comercio territorial, nacional como internacional, son identidades propias y abiertas que tienden a descentralizar las vías de comercio, según Lira (2003) en esta fase de globalizaciones recién los territorios podrán participar una vez cuando consigan entender que la adaptación a las transformaciones que produce sobre la estructura productiva con base en el conocimiento y sus aplicaciones bien entendidas hacia los sectores implicados. Esto implica desarrollar bien las habilidades para producir ventajas o capacidades para construir sobre si mismos sectores potenciales de ser inseridos en el comercio, nacional e internacional.

El avanzo de acuerdo al ámbito político, cultural, permite observar con clareza el nivel que se requiere para poder competir entre regiones que comparten un mismo objetivo. La falta de participación de los agentes sociales anulara todo tipo de acuerdo futuro, colocando en un plano inestable los intereses comprometidos. El grado de participación dará posibilidades a elaborar un plan propio que se ajuste a las demandas de cada territorio, en vista a que este último al descentralizar las políticas gubernamentales, logra estimular el desarrollo extensivo y adaptar normativas que caracterizan a cada región. De cierta forma estas normas solo se expresan cuando se da espacio para el envolvimiento ciudadano y lograr su compromiso empático por la región. Cuando se guardan intereses comunes de dependencia que por su vez genera proyectos que generan resultados positivos que permiten crear, desarrollar y fortalecer las unidades productivas por medio acción 
participativa con capacidad para enfrentar los retos que se configuran en los ámbitos de desarrollo local. El desafío que se produce en el momento que estos eventos dejan de presentarse es escalar la naturaleza en categorías organizacionales. Establecer medidas de ajuste como el desarrollo de protocolos como sistema de análisis y que evaluación de acciones, que den ventajas para aquellas capacidades que los agentes sociales.

\section{El papel de los excedentes en el desarrollo económico.}

El carácter estratégico que genera crecimiento a través del retorno de los excedentes de producción es un factor que toma como base de amplitud que utiliza como acumulo de productividad (BETTELHEM, 1976). Es el excedente de producción agrícola que a pesar de los obstáculos que pueden surgir, desempeñan un rol estratégico para el desarrollo territorial ya que esta íntimamente vinculado al sector industrial como motor propulsor para establecimiento de programas de desarrollo sectoriales. Se entiende que la existencia del excedente es una condición necesaria para el desarrollo y crecimiento de una sociedad, es interesante destacar que esto no es suficiente para alcanzarlo pero por menos es satisfactorio. El concepto aquí colocado se refiere al tipo de excedente cuando es utilizado como medio de financiamiento al producir mejoras a la capacidad de producción. Consecuentemente el efecto que produce es el aumento de la calidad de vida de los trabajadores, al realizar nuevas inversiones productivas y las inversiones indirectas utilizadas para el aumento de la capacidad en la infraestructura local, como ejemplo direccionarlo hacia instituciones públicas, escuelas, hospitales, servicios de vivienda, al aumentar la capacidad disponible de los servicios públicos.

Cuando el excedente económico se torna efectivo entre el producto social de una comunidad y su efectivo consumo, produce un tipo de ahorro o acumulación de activos que se materializan en tangibles de varias especies, durante un cierto periodo de tiempo, considerando bienes de equipamientos tecnológicos, unidades productivas, etc. Las formaciones económicas generan por si solo excedentes económicos que se materializan en efectivo de estructura y magnitud en cuanto al valor de existencia económica en la fase del desarrollo. El excedente económico potencial es la diferencia entre el producto social que se extrae de un medio natural o tecnológico con el auxilio de recursos productivos, reorganizando los medio de producción y distribución del producto social. Esto indica realizar profundos cambios en la estructura social, Baran ( 1977) destaca cuatro tipos de excedente productivo nefastos pero necesarios para el desarrollo en una economía capitalista: i) consumo superfluo, ii) la producción que deja de ser efectiva frente a utilización de trabajadores no productivos, iii) producción que se pierde, debido a una organización irracional propensa al desperdicio, iv) producción que se obtiene a causa de existir el desempleo sin que pase por el desajuste desordenado de la producción y la deficiencia de la búsqueda efectiva.

El excedente económico planificado de una economía global en una sociedad, suma a la diferencia entre el producto social "optimo" esto según Baran (1977) la sociedad puede realizar en un ambiente natural tecnológico. La dificultad enorme es distribuir proporcionalmente a los medios de producción el capital o sea los excedentes como forma de reposición (SRAFFA, 1977).

El aspecto estratégico de identificar los excedentes productivos como demarcador de criterios para hospedar indicadores de resultados para los territorios o regiones dispuestos al desarrollo local. Sirven como instrumento valido de coexistencia de las entidades sociales con sus planos programáticos de estimular la participación en los procesos productivos de inserción regional al mercado comercial. Los indicadores son de bastante utilidad visto que su práctica mensura los resultados por los excedentes a ser aplicados. El estudio de las regiones que están habilitadas y dispuestas a desenvolver formaciones productivas, esto significa comprometer las cadenas en un ciclo permanente para la obtención de resultados futuros. La utilidad de aquellas regiones dispuestas a participar en el diseño de formación productiva, como medio de transformación de sus bases de producción. Se direccionan para encadenamientos dinámicos entre los grupos sociales que se aplican en microrregiones que poseen un mínimo de capacidad productiva y donde exista un conglomerado o redes, formaciones de productores consolidados, que establecen concomitantemente cooperación y creación de alianzas estratégicas de trabajo. Aquellos conglomerados productivos, pequeños 
industriales, pesqueras artesanales, asociaciones de servicios y oficios, artesanos, asociaciones agrícolas y otras formas de sociedades que generen activos y se inserten en la cadena productiva.

Para tal efecto, el proceso de transformación lleva consigo un elemento en común a todos y excluyente por su forma de actuar. La incorporación de instrumentos innovadores que incrementen y produzcan satisfacción por los cambios al proceso transformador. Se pretende establecer una relación de las redes de agrupaciones con los lazos que este vinculo genera, lazos que producen un "capital social relacional", Vale (2006) ya preexistente en anteriores emprendimientos que funcione como plataforma para lanzamiento de otras nuevas empresas. Al observar esta relación se crean activos que producen utilidades proporcionales al tamaño y al tipo de los lazos.

Sin embargo, aquellos vínculos de "capital social relacional" a medida que se consolidan estimulan la formación de sucesiva plataformas, proporcionando naturalmente un motor para nuevas relaciones. El beneficio tanto por las entidades como en los miembros individuales, para la creación de nuevos emprendimientos genera un encadenamiento continuo. Los indicadores (Figura 3) que satisfacen esta relación para alcanzar resultados locales están relacionado de acuerdo a una investigación de campo en la región de Ubá, Brasil realizado (VALE, 2006, p.38).

Figura 3

\section{Indicadores que satisfacen la relación, desempeño, innovación, lazos y dinámica territorial.}

\begin{tabular}{l|l}
\hline Instrumentos & \multicolumn{1}{c}{ Situación de los lazos sociales } \\
$\begin{array}{l}\text { Densidad de red } \\
\text { Amplitud }\end{array}$ & $\begin{array}{l}\text { Mide el grado de conectividad de una red en un determinado momento, a partir de la afeirção } \\
\text { de frecuencia de contactos } \\
\text { Mide el número de diferentes contactos existentes en una determinada red, incluyendo contactos } \\
\text { Locales y Regionales. }\end{array}$ \\
\hline $\begin{array}{l}\text { Densidad } \\
\text { Enraizamiento } \\
\text { Sectorial }\end{array}$ & $\begin{array}{l}\text { Mide la alteración en la intensidad de contacto dentro de un misma categoría de contacto } \\
\text { en un determinado periodo de tiempo }\end{array}$ \\
\hline $\begin{array}{l}\text { Mesempeño } \\
\text { Empresarial }\end{array}$ & $\begin{array}{l}\text { Mide el encadenamiento familiar de la actividad (generaciones), empleo en nuevas empresas. } \\
\text { de empresas }\end{array}$ \\
\hline
\end{tabular}

Fuente: Revista Desenvolvimento Econômico: Adaptado de VALE (2006).

Para tal efecto, la activación de los instrumentos solo es susceptible cuando el crecimiento económico es estimulado por el ciclo virtuoso de aplicaciones de capacidad de generar ahorro y que estos sean destinadas a la creación de capital social. Esto significa que "no solo importan los márgenes de ahorro sino también es primordial resolver acerca del destino de ese ahorro" (PINTO y SANTA CRUZ, 2006, p.5).

\section{La capacidad de las regiones en la mejora del desarrollo territorial}

El desarrollo de las regiones a través de sus programas de mejoramiento regional en cuanto al ámbito de competitividad, visa evaluar según sus formaciones productivas por medio de agencias regionales. Acompañar las articulaciones y sus efectos locales por el apoyo productivo de los organismos públicos según 
las necesidades de cada entidad. Las respuestas a las transformaciones sucesivas sobre su desempeño concreto, se ve de inmediato en los logros y alcances obtenidos, véase el caso de los programas establecidos en regiones de Europa LEADER, de Latino América, EXPIDER como experiencia piloto del BID, PRODEM en la regiones del nordeste brasilero con apoyo del Banco del Nordeste, los programas MiPyME por las agencias de desarrollo locales de financiamiento vía CORFO en Chile, las Mancomunales en el altiplano Boliviano. Esto son transformaciones productivas junto a los cambios de un desarrollo institucional. Promover según las evaluaciones el desarrollo sostenible regional como guía de análisis y observación a estas mudanzas un proyecto territorial compartido, que reconozca la diversidad de territorios. Planificar el desarrollo de regiones que lleven a distinguirlas como modelos, con la finalidad de establecer niveles que alcancen un desarrollo pleno a medio y largo plazo. Considerándose por último, varios aspectos relativos a los objetivos que las políticas de participación social decidan establecer con la ayuda de organizaciones. Dando formación y fortalecimiento a organizaciones emprendedoras, pequeñas y medianas empresas, que posean una visión moderna y real de la situación, reforzando las sinergias publicas-privadas. También que las regiones estén en sintonía con sus potencialidades en cuanto a sus recursos disponibles Lima (2007). Considerando también la integración para esta vía por otras regiones y para sus futuras generaciones.

Esta capacidad que las regiones poseen se visualiza en la práctica gracias a las políticas de desarrollo económico que cada grupo social aplica en sus propios territorios. Aspectos relativos al diseño de un programa regional que con base en indicadores estructurales y con dimensiones operacionales con características i) sociales, ii) ambientales, iii) económicas, iv) institucionales. Al implementar estos elementos en una dinámica de actuación conjunta solo es posible al crear efectos sinérgicos que sean capaces de estimular en presencia de sistemas que se auto-fortalezcan. Los ordenamientos territoriales que muestran capacidad de generar activos en sectores ignorados últimamente y tratados separadamente como si no existieran lazos de congruencia, sectores agrarios en las regiones rurales, se han reorientado con mayor índice a fortalecer los lazos entre las asociaciones cooperadas, formando conglomerados de pequeña escala, que participan del proceso productivo del cual hacen parte (Figura.4).

Figura 4

\section{Cuadro de Relaciones entre Tipos y Aspectos de explotaciones económicas}

\begin{tabular}{|c|c|}
\hline Tipos & Aspecto de las explotaciones agrícolas \\
\hline $\begin{array}{l}\text { Explotaciones campesinas } \\
\text { subsistencia }\end{array}$ & Minifundio, producción auto consumo, diversificación de actividades \\
\hline Explotaciones & \\
\hline familiares & $\begin{array}{l}\text { Tamaño varia según el país o región, utiliza mano de obra, producción para autoconsumo, } \\
\text { con excedentes para el mercado }\end{array}$ \\
\hline Pequeña agricultura & Tamaño varia según el país o región, utiliza mano de obra familiar asalariada, orienta \\
\hline Comercial en transición & $\begin{array}{l}\text { la producción al mercado, especializa la producción, poseen activos y capacidad para } \\
\text { el potencial empresarial }\end{array}$ \\
\hline $\begin{array}{l}\text { Agricultura empresarial } \\
\text { capitalista }\end{array}$ & $\begin{array}{l}\text { Emplea mano de obra asalariada permanente, incorpora técnicas modemas de producción, } \\
\text { |alto nivel de productividad y con elevada integración hacia el mercado }\end{array}$ \\
\hline
\end{tabular}

Fuente: elaborado por el autor. 
El conjunto de elementos que hacen parte de la fuerza de trabajo que integran las políticas sociales de desarrollo, se elaboran de acuerdo a una relación de inquietudes que según propuestas formulan una serie de interrogantes sobre ¿Cuales serán las condiciones y desafíos propuestos? ¿El clima regional facilita el desarrollo sostenible? ¿La presión competitiva y las expansiones subyacentes en sus proporciones, recompensa la innovación y el dinamismo productivo? ¿Si fueran limitados, estos serian dirigidos para los sectores de la economía donde podrían ser más productivos junto a la infra-estructura adecuada a sustentar las tasas de crecimiento local? ¿Es clara la política de precios que siendo competitiva con relación al carácter de crecimiento es equitativo con las demás regiones?

Los recursos disponibles localizados en las regiones deben ser utilizados con la mayor eficiencia, adaptándolos a directrices que establezcan una amplitud en los mercados negociables y que agreguen un valor sustancial a los productos comercializados, garantizando el excedente de inversión manteniendo el padrón y racionalización de los recursos. Puesto que algunas regiones se destacan por mantener acuerdos productivos, sin embargo, con mercados internos reducidos, lo que seria un alto riesgo reflejo de las desventajas frente a las otras regiones. Lo que es claro es dar garantías a los agentes sociales por medio de especializaciones, mano de obra calificada. El correcto y debido uso de las directrices que se establezcan para el buen funcionamiento de los recursos genera una estrategia saludable que se adecua a economías locales de regiones de Latino América.

Desde luego al exponer estas directrices se localizan algunos problemas en regiones con elevada producción industrial, concomitante en territorios con altas tasas de desempleo. Al final el objetivo de las políticas regionales al presentarse estos escenarios es formular acciones que no sean "parches". Por el contrario, sino que, buscar dentro de las regiones sectores económicos que posean base para un crecimiento viable como auto-sostenible Shanks (1973), este desarrollo regional no debe ser estimado a corto plazo, ni menos concentrarse en eliminar los "inchazos" aislados de desempleo. Como en todo los procesos mal diseñados, se ignoran las reales necesidades permanentes de las industrias, las cuales deban se establecer donde puedan operar con el máximo de eficiencia. Lo que se pretende es la creación de un plano regional equilibrado para el desarrollo, estableciendo un nexo entre necesidades a recursos y los requisitos y recursos de cada región.

Ya el desarrollo solo es posible y efectivo una vez que tanto las dependencias como las estructuras de arriba hacia abajo en un proceso descentralizador se depriman y anulen. Para lograrlo se requiere una propuesta robusta que considere que las regiones consigan en periodos estables la auto-suficiencia (SOUZA, 1999). Para lograrlo según el autor deben incorporarse instrumentos de permitan el espontáneo desarrollo de sus propias economías, solo es posible, cuando son factibles los instrumentos financieros y dotados de capacidad y estructura para dar soporte al peso a largo plazo (GIAMBIAGI, 2005). Desde luego al desarrollo debe ir acompañado de restos que deban ser evaluados constantemente. Neto (2005), coincide en este posible largo plazo estableciendo una economía "ambiental" de estabilidad macroeconómica, lo que implica i) niveles de inflación que sean bajos y previsibles, ii) adoptar una política fiscal estable y responsable, iii) tasas de interés real, de cambio real de equilibrio previsible, que otorgue garantías para una balanza en cuenta corriente sostenible a largo plazo, iv) amplia apertura comercial y con tasas de crecimiento del PIB cerca del pleno empleo, v) un sistema financiero que sea bien regulado y sólido. En tanto que la relación de los elementos macroeconómicos genera estas condiciones no es seguro saber exactamente como obtenerlo.

\section{El proceso regional de autogestión como polo de desarrollo}

La localización tangible en un espacio geográfico viable, genera líneas de acción estratégicos para establecimientos de sectores productivos que complementan las actividades que les subyacen, dando origen a la construcción de sistemas urbanos. Siendo que es permisible comentar que el crecimiento no surge simultáneamente en todas las regiones, a la vez, surgen polos sectoriales de crecimiento como grados de diversa intensidad (PERROUX, 1967). Probablemente esta premisa sirva de referencia para resolver un plan que pretenda crear instituciones económicas con fuertes lazos de participación social. Instituciones con 
estructuras dinámicas y auto-suficientes en cuanto a la obtención de recursos y que sea generadora de políticas que impulsen estrategias innovadoras para el desarrollo de las entidades locales, formulen sinergias. Ausente de pretensiones de agencias de gobierno y centralistas en cuanto a políticas de financiamiento, esto anula la total regulación por parte de agencias centrales de gobierno. Esta reorientación implica al mismo tiempo una nueva concepción del espacio organizacional de las políticas de integración regional. Pues la formulación de políticas de crecimiento para regiones son auto-determinadas por ellas mismas y sus territorios en conjunto, deseando un cambio en la visión conservadora de las autoridades centralizadoras y preferencialmente partidistas, cual siempre impone los intereses particulares en desmedro de las reales necesidades de los agentes sociales y sus territorios.

Esto es resultado de la observación que se obtiene por el reiterado enfoque que las entidades gubernamentales dan al mismo problema, usando siempre el mismo prisma de observación pre-definido. Implica decir que las autoridades públicas mensuran en ausencia de una dinámica regional. Lo que a simple vista se observa, según el autor es un horizonte con espacios limitados en una línea recta en cuanto a los programa de desarrollo regional, con la finalidad de incluir recursos potenciales todavía no explorados. La situación que da expansión a espacios económicos interdisciplinares es el desarrollo de estímulos a procesos regionales en función de las disposiciones técnicas que produzcan habilidades para sectores de mayor interés y que estén relacionados por determinadas condiciones funcionales (PAELINCK, 1977).

Según el estudio de "Dinámica económica de la región de Liege" que redefine la concepción de las ventajas de la polarización regional sobre la visión integrada funcional por la capacidad técnica, que debido al flujo de productos y la generación de rentas, produce condiciones de expansión del crecimiento a empresas técnicamente vinculadas. Determina prosperidad para sectores económicos por medio de rentas (polarización de rentas), con el fin de producir un aumento del salario regional, a causa de la concentración de emprendimientos en localidades determinadas. Las relaciones entre el espacio y el aprovechamiento local de los recursos disponibles pendiente de las preocupaciones de crecimiento, lanzan un eje conductor para la formulación de programas de auto-gestión. A principio es el proceso de desarrollo en espacios que interesan de inmediato al crecimiento y no este que sirva como apenas un instrumento al servicio del desarrollo, sino que como un objetivo en si (LOPES, 2001).

\section{Consideraciones finales}

La falta de incentivos necesarios al ahorro e inversión ineficiente tanto a nivel nacional como extranjero; el excesivo papel como a la vez sofocante rol del Estado adjunto a sus agencias reguladoras y la debilitación concomitante del sector financiero privado con sus políticas proteccionistas. Una suma de obstáculos que impiden el crecimiento que subyace al desarrollo de políticas ciudadanas locales. Las estrategias propuestas cumplen evaluar la capacidad que poseen cierta regiones de América Latina, a examinar sus problemas de forma constructiva (BELA et al., 1986).

Al plantear este trabajo sobre el uso de instrumentos que fortalecen el poder negociador al observar experiencias próximas y siendo motivadoras todavía más, aun continúan siendo efímeras por la débil profundidad de penetración en espacio periféricos sea falta de medio comunicacionales o mismo herramientas tecnológicas que las hagan ver.

Desarticular las políticas que los agentes económicos partidistas elaboran para beneficios propios, pues la descentralización solo vendrá cuando entiendan por vía resultados efectivos que las directrices son guiadas por el peso de protagonismo que representan en las regiones, la sociedad civil y el poder público local.

Las matrices propuestas por medio de proyectos estratégicos de desarrollo, fortalezcan la estructura de las cadenas productiva, viabilicen los programas de integración orientado los territorios hacia la formación de 
polos regionales. Medidas que deben considerar la participación espontánea de los grupos sociales visando el trabajo que las transformaciones demandan.

La competitividad concebida por las regiones solo será efectiva cuando se observen de forma sistémica que no es solo un atributo que por ella se llega (SHEJTMAN y BERDEGUE, 2002). Si bien que por medio de elementos que acompañen el proceso integrado y que sus sinergias otorguen características tecnológicas, financieras, pedagógicas, investigativas, desarrollo, o sea una multidisciplinaria fusión de herramientas de apoyo.

\section{Referencias}

BARAN, P. A economia política do desenvolvimento. Rio de Janeiro: ZAHAR EDITORES, 1977.

BARROSO, P, V. Competencia territorial y su relación con los ámbitos de competitividad en la perspectiva de desarrollo regional y local: Su implicación en la investigación en la zona poniente del Estado In: ANALES DE CONFERENCIA INTERNACIONAL ESTRATEGIA DE DESARROLLO Y ALTERNATIVAS PARA AMÉRICA LATINA Y EL CARIBE, p.1-30, 18 de octubre de 2006, Puebla, México.

BETTELHEM, C. Planificação e crescimento acelerado. 2. ed. Rio de Janeiro: Zahar Editores, 1976.

CHOSSUDOVSKY, M. The globalization of poverty: Impacts of IMF and world bank reforms. Penang, Malaysua: Third World Network, 1997.

DELFIM NETTO, A. Desenvolvimento segundo a CEPAL e o desenvolvimento segundo o Brasil, Rio de Janeiro: Elsevier, 2005, p.225-256.

ELIAS, N. O processo civilizador: do controle social ao autocontrole. v. 2. Rio de Janeiro: Zahar Editores, 1993.

ESTRELLA, R; VALERIO, V. Inteligencia competitiva regional para impulsar el desarrollo local-endógeno. In: Anales Conferencia Internacional Estrategia de Desarrollo y Alternativas para América Latina y el Caribe, 17 de octubre de 2006, Puebla, México, p. 3-37.

GARDNER, B; MOORE, D. Relações humanas na indústria: Comportamento organizacional e administrativo. 2. ed. São Paulo: ATLAS S.A, 1969.

GIAMBIAGI, F, et al. Economia brasileira contemporânea: O financiamento do desenvolvimento, Rio de Janeiro: Elsevier, 2005, p.335-355.

HIRSCH, F. Limites sociais do crescimento. Rio de Janeiro: ZAHAR EDITORES, 1979.

LIMA, F. Indicadores para analisar e promover o desenvolvimento sustentável regional. Revista de Economia \& Relações Internacionais, São Paulo, v. 5, n. 10, jan., 2007, p. 43-58.

LIRA, L. Disparidad, competitividad territorial y desarrollo local y regional en America Latina. Revista CEPAL, Serie Gestión Pública, n. 33, abr., Santiago de Chile, 2003.

LOPES, S. Desenvolvimento regional: Problemática, teoria, modelos. 5. ed. Lisboa: Gulbenkian, 2001.

LORENTE, L. Estado y sector privado: Dónde trazar los limites? Portafolio. Análisis, Revista CEGES, abr. 2006, Colombia.

MARTINEZ, A. Introducción al análisis de proyectos de inversión. México: Trillas: ESAHE, 1993. 
OBSERVATORIO EUROPEO. La competitividad territorial: Construir una estrategia de desarrollo territorial con base en la experiencia Leader. Observatorio Europeo LEADER, "Innovación en el medio rural”, Fascículo 1, dic. 1999, p. 5-43.

PAELINCK, J. A teoria do desenvolvimento regional polarizado. In: SCHARTZMAN, J. Economia regional. Belo Horizonte, CEDEPLAN, 1977, p. 157-174.

PERROUX, F. A economia do século XX. Lisboa: HERDER, 1967.

PINTO SANTA CRUZ, A. El financiamiento del desarrollo y el círculo vicioso de la pobreza. Revista Desenvolvimento Econômico, Salvador, Ano VIII, n. 13, jan. 2006.

ROSSETI, J. Política e programação econômica. 7. ed. São Paulo: ATLAS, 1987.

SCHEJTMAN, A; BERDEGUÉ, J. A. Desarrollo Territorial Rural. Santiago do Chile: RIMISP, 2002. p. 10-55.

(Documento preparado para o FIDA e BID).

SHANKS, M. A economia da tecnologia: Os inovadores. São Paulo: ATLAS, 1973.

SOUZA, J. Desenvolvimento econômico. 4. ed. São Paulo: ATLAS, 1999.

SRAFFA, P. Produção de mercadorias por médio de mercadorias: Prelúdio a uma nova crítica da teoria econômica. Rio de Janeiro: ZAHAR, 1977. p. 22-33.

VALE, G, M, V. Laços como ativos territoriais: Uma nova abordagem para desenvolvimento regional. Revista Desenvolvimento Econômico, Salvador, Ano VIII, n. 14, julho 2006, p. 34-40. 